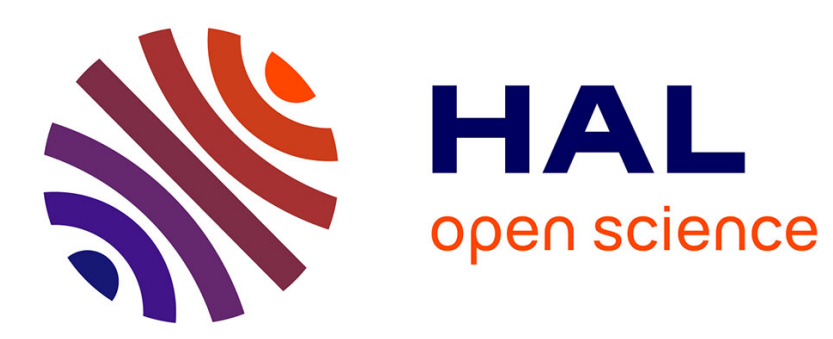

\title{
Identification de gènes de restauration de fertilité sur cytoplasmes stérilisants chez le tournesol
}

\author{
Patrice Leclercq, J. P. Philippon
}

\section{To cite this version:}

Patrice Leclercq, J. P. Philippon. Identification de gènes de restauration de fertilité sur cytoplasmes stérilisants chez le tournesol. Agronomie, 1984, 4 (6), pp.573-576. hal-00884674

\section{HAL Id: hal-00884674 \\ https://hal.science/hal-00884674}

Submitted on 1 Jan 1984

HAL is a multi-disciplinary open access archive for the deposit and dissemination of scientific research documents, whether they are published or not. The documents may come from teaching and research institutions in France or abroad, or from public or private research centers.
L'archive ouverte pluridisciplinaire HAL, est destinée au dépôt et à la diffusion de documents scientifiques de niveau recherche, publiés ou non, émanant des établissements d'enseignement et de recherche français ou étrangers, des laboratoires publics ou privés. 


\title{
Identification de gènes de restauration de fertilité sur cytoplasmes stérilisants chez le tournesol.
}

\author{
Patrice LECLERCQ \\ avec la collaboration technique de J. P. PHILIPPon (Promosol) \\ I.N.R.A., Station d'Amélioration des Plantes, F 63039 Clermont-Ferrand Cedex
}

RÉSUMÉ Il semble qu'on ait, pour la restauration de la stérilité mâle française chez le tournesol, un système de 2 gènes
dominants complémentaires. La plupart des restaureurs de fertilité, peut-être même tous, sont porteurs des
gènes (Rf1 + Rf2). La plupart des mâle-stériles classiques sont porteurs d'1 gène (Rf2).
La stérilité mâle Kouban semble restaurée selon les mêmes règles, mais nous supposons que les 2 dominants
complémentaires, Rk1 et Rk2 sont différents des gènes de restauration français, Rf1 et Rf 2 .

Mots clés additionnels : Helianthus petiolaris, Helianthus lenticularis, stérilité mâle.

Additional key words : Helianthus petiolaris, Helianthus lenticularis, male sterility.

\section{INTRODUCTION}

Si on reconnaît l'existence d'une stérilité mâle cytoplasmique à partir du moment où on a observé 2 descendances successives entièrement mâle-stériles dans une même filiation maternelle et si on distingue un cytoplasme stérilisant (c'est-à-dire une source de stérilité mâle) des autres cytoplasmes par l'éventail des restaureurs et mainteneurs, alors on connaît actuellement 2 cytoplasmes stérilisants chez le tournesol : le français (I.N.R.A.) et le soviétique (Kouban), (LECLERCQ, 1983).

La distinction des cytoplasmes par le seul éventail des restaureurs peut prêter à critique. Ainsi, dans le cas du cytoplasme français (I.N.R.A.), on a mis en évidence 2 gènes complémentaires de restauration : $\mathrm{Rf}_{1}$ et $\mathrm{Rf}_{2}$. Si l'on dispose d'une lignée $F \mathrm{Rf}_{1} \mathrm{Rf}_{1} \mathrm{rf}_{2}$ $\mathrm{rf}_{2}$, elle apparaîtra restauratrice en présence d'un mâle-stérile $\mathrm{S} \mathrm{rf}_{1} \mathrm{rf}_{1} \mathbf{R f}_{2} \mathrm{Rf}_{2}$ et mainteneuse en présence d'un mâle-stérile $\mathrm{S} \mathrm{rf}_{1} \mathrm{rf}_{1} \mathrm{rf}_{2} \mathrm{rf}_{2}$. Dans un tel cas, il pourra paraître tentant de proclamer l'existence d'un nouveau type de cytoplasme, puisque le $2^{\mathrm{e}}$ mâle- stérile n'a pas exactement les mêmes restaureurs que le $1^{\text {er }}$. Mais cette conclusion serait fausse.

Pour éviter de telles confusions, il est important d'identifier, au moins sur quelques lignées, les gènes de restauration mis en jeu. C'est le but de la présente étude.

\section{REVUE BIBLIOGRAPHIQUE COMMENTÉE}

La stérilité mâle cytoplasmique française (I.N.R.A.) a fait l'objet d'une publication en 1969 (LECLERCQ, 1969). En $R_{1}(F 1 \times$ parent mâle tournesol), en même temps que nous découvrions les premières plantes mâle-stériles, leurs sœurs mâle-fertiles étaient vraisemblablement porteuses des gènes de restauration. En effet, la fécondation d'Helianthus petiolaris (S Rf Rf) par le tournesol ( $F \mathrm{rf}$ rf) nous a donné une F1 mâle-fertile ( $\mathrm{S}$ Rf $\mathrm{rf}$ ). Cette $\mathrm{F}$ 1, fécondée par du tournesol ( $\mathrm{F}$ rf rf) a produit logiquement, à la fois des plantes mâle-stériles (S rf rf) et mâle-fertiles ( $\mathrm{R}$ R rf). Une opinion a été émise par certains com- 
mentateurs (VRANCEANU \& STOENESCU, 1971) selon laquelle la stérilité mâle cytoplasmique a été découverte en France et les premiers gènes de restauration de fertilité ont été reconnus à l'étranger (Etats-Unis, Roumanie). Cette opinion ne résiste pas à l'examen : en effet, par la logique interne du système, en R1 nous avons mis en évidence en même temps la stérilité mâle cytoplasmique et les gènes de restauration de fertilité, que nous avons appelés $\mathrm{Msc}_{1}$, qui provenaient du croisement réalisé à Clermont-Ferrand, évoqué cidessus, entre $H$. petiolaris et $H$. annuus (tournesol). L'existence de gènes de restauration de fertilité chez $H$. petiolaris découle logiquement de l'interprétation théorique ci-dessus $(H$. petiolaris $=\mathrm{S} \mathrm{Rf} \mathrm{Rf})$ mais, de plus, nous l'avons prouvée directement en fécondant un mâle-stérile par $\boldsymbol{H}$. petiolaris. Nous avons alors établi que $H$. petiolaris ne différait des mâle-stériles utilisés que par un gène dominant de restauration de fertilité (LECLERCQ, 1979).

ENNS et al. (1970) signalent l'existence d'un gène dominant de restauration de fertilité à partir de plusieurs sources américaines dont l'une vient de H. petiolaris.

KINMAN (1970) reconnaît l'existence d'un gène dominant de restauration de fertilité qu'il appelle $\mathrm{Rf}_{1}$.

VRANCEANU \& STOENESCU (1971) signalent la rareté des gènes de restauration chez le tournesol mais trouvent cependant, dans une seule lignée, un gène de restauration qu'ils dénomment, provisoirement, $\mathrm{Rf}_{2}$, pour le distinguer du $\mathrm{Rf}_{1}$ de KINMAN, sans que la différence entre $\mathrm{Rf} 1$ et $\mathrm{Rf} 2$ ait été mise en évidence.

A ce stade des connaissances, on peut abandonner la désignation $\mathrm{Msc}_{1}$ (LECLERCQ, 1971) et rejoindre la dénomination de KINMAN : $\mathrm{Rf}_{1}$. Les gènes dominants découverts par LECLERCQ, ENNS et VRANCEANU, pourraient être appelés provisoirement et respectivement : $\mathrm{Rf}_{\mathrm{L}}, \mathrm{Rf}_{\mathrm{E}}, \mathrm{Rf}_{\mathrm{V}}$; l'affectation d'un $\mathrm{n}^{\circ}\left(\mathrm{Rf}_{2}\right.$ par exemple) ne devrait se faire que si on a prouvé que $\mathrm{Rf}_{1}$ et $\mathrm{Rf}_{2}$ sont différents.

Par la suite (FICK \& ZIMMER, 1974), a été mise en avant l'hypothèse de 2 gènes dominants complémentaires, $R f_{1}$ et $R_{2}$, expliquant en $F 2$ une ségrégation de 9 mâle-fertiles pour 7 mâle-stériles. La plupart des mâle-stériles possédaient le gène $\mathrm{Rf}_{2}$ seulement et la plupart des restaureurs, les 2 gènes $\mathrm{Rf}_{1}$ et $\mathrm{Rf}_{2}$ : dans ce cas, on observe en $\mathrm{F} 2$ la disjonction pour un seul gène $(3: 1)$. Cependant, en croisant le mâle-stérile Ha 267 par le restaureur Rha 280 , on trouve $9: 7$ en F2. Les auteurs mettent en avant 2 hypothèses exclusives l'une de l'autre :

- Rha 280 a $\mathrm{Rf}_{1}$ seulement et $\mathrm{Ha} 267, \mathrm{Rf}_{2}$ seulement,

- Rha 280 a $\mathrm{Rf}_{1}$ et $\mathrm{Rf}_{2}$ et $\mathrm{Ha} 267$ n'a aucun gène dominant de restauration.

Ils ne choisissent pas entre ces 2 hypothèses.

GIMENEZ \& FICK (1975) mettent en avant l'hypothèse de 4 gènes pour expliquer restauration et fertilité. La restauration serait assurée si au moins 2 gènes dominants sont présents ensemble. Mais les résultats chiffrés à l'appui de cette hypothèse sont peu nombreux.

L'un des gènes de restauration serait présent dans Ha 89.

En Inde, REDDY \& THAMMI RAJU (1977) mettent en évidence, en $\mathrm{F} 2$ et $\mathrm{F} 3$, l'existence de 2 gènes domi- nants complémentaires $(9: 7$ en $F 2)$ à partir d'un matériel venu de France. Les auteurs écrivent : « la lignée mâle stérile était évidemment un double récessif et le mâle un double homozygote dominant ". Mais cette conclusion n'est pas évidente du tout, car l'attribution à chacun des 2 parents d'un seul gène dominant (par exemple $\mathrm{Rf}_{1}$ pour le mâle, $\mathrm{Rf}_{2}$ pour la femelle) donnerait en $F 1$ et $F 2$ exactement les mêmes résultats.

En 1978, VRANCEANU \& STOENESCU confirment l'existence, selon les croisements mis en jeu, d'un gène dominant de restauration, ou de 2 ou de 3 gènes dominants complémentaires.

En 1979, LECLERCQ confirme l'hypothèse de 2 gènes dominants complémentaires : à partir de $2 \mathrm{~F} 1$ ayant un parent mâle commun, Bza1, on obtient 2 F2 dont l'une donne 1 stérile pour 3 fertiles (CIC $61 \times$ Bza1), l'autre 7 stériles pour 9 fertiles (CA $4 \times$ Bza1). Comme Bzal a au moins 1 gène de restauration de fertilité (soit Rf1, pour suivre la terminologie de KINMAN), on doit admettre qu'il en a 2, Rf1 et Rf2. En effet, si Bzal n'avait qu'un seul gène dominant de restauration (Rf1), aucune formule ne conviendrait pour CIC 61 mâle-stérile :

- $S \mathrm{rf}_{1} \mathrm{rf}_{1} \mathrm{rf}_{2} \mathrm{rf}_{2}$ et $\mathrm{S} \mathrm{Rf}_{1} \mathrm{Rf}_{1} \mathrm{rf}_{2} \mathrm{rf}_{2}$ ne donneraient pas de mâle-fertiles en $F 1$,

- $\mathrm{S} \mathrm{rf}_{1} \mathrm{rf}_{1} \mathrm{Rf}_{2} \mathrm{Rf}_{2}$ donnerait $7: 9$ en $\mathrm{F} 2$,

- $\mathrm{S} \mathrm{Rf}_{1} \mathrm{Rf}_{1} \mathrm{Rf}_{2} \mathrm{Rf}_{2}$ ne serait pas mâle-stérile.

Ainsi Bzal est de génotype Rf1 Rf1 Rf2 Rf2.

Comme CA $4 \times$ Bzal donne en $\mathrm{F} 2$ une ségrégation $7: 9$, correspondant à la disjonction de 2 dominants complémentaires, on doit admettre pour CA 4 la formule S rf1 rf1 rf2 rf2, c'est-à-dire 0 gène dominant de restauration.

D'une façon générale, chaque fois qu'on observe une F1 mâle-fertile et une F2 ayant 1 mâle-stérile pour 3 mâle-fertiles, on est amené à l'hypothèse de 2 dominants chez le parent mâle ( $\mathrm{Rf} 1+\mathrm{Rf} 2)$ et d' 1 dominant chez le parent femelle ( $\mathrm{R} f 2$ en règle générale), à partir du raisonnement tenu pour CIC $61 \times$ Bazl.

\section{RÉSULTATS NOUVEAUX}

Nous avons voulu vérifier, en 1981, la ségrégation 7 : 9 pour la F2 de « Relax » $($ CA $4 \times$ Bza 1).

Alors qu'en 1979 on avait 21 mâle-stériles pour 31 mâle-fertiles, on observe, en 1981, 104 mâle-stériles pour 115 mâle-fertiles; comme il n'y a pas de différence statistique entre les 2 années $\left(\chi^{2}=0,59\right)$, on peut les additionner, ce qui donne $125 \mathrm{~ms}$ et $146 \mathrm{mf}$, en accord avec la théorie $7: 9\left(\chi^{2}=0,53\right)$. Mais la plupart des $\mathrm{F} 2$ montrent $1 \mathrm{~ms}$ pour $3 \mathrm{mf}$, suivant le tableau 1 , ce qui conduit à attribuer 2 gènes dominants (Rf1 et $R$ f2) au parent mâle, un seul (Rf2) au parent femelle.

Seule la $\mathrm{F} 2$ de $\mathrm{CC} 40 \times \mathrm{PAC} 1$ échappe à la règle $1: 3$. Dans ce cas, comme CC 40 peut se voir attribuer la formule S rf1 rf1 Rf2 Rf2 (d'après CC $40 \times$ PAC 2, observé en $\mathrm{F} 2$ avec $1: 3$ ), on est conduit à donner à PAC 1 la formule Rf1 Rf1 rf2 rf2. Comme on avait conclu que CA 4 avait 0 gène dominant, l'hybride CA $4 \times$ PAC 1 devait être mâle-stérile : or, ce n'est pas le cas, puisqu'il montre $3 \mathrm{~ms}+44 \mathrm{mf}$, en 1982 , $0 \mathrm{~ms}+12 \mathrm{mf}$, en 1983. Il subsiste ici un mystère. 
TABLEAU 1

Observations de la stérilité mâle dans des descendances $F 2$ d'hybrides fabriqués avec des lignées mâle-stériles sur le cytoplasme $S$ (français). Male sterility in $\mathrm{F} 2$ progenies of hybrids made with male sterile lines with S cytoplasm (French).

\begin{tabular}{|c|c|c|c|c|}
\hline \multirow[b]{2}{*}{ Nom de l'hybride } & \multirow[b]{2}{*}{$\mathrm{ms}$} & \multirow[b]{2}{*}{$\mathrm{mf}$} & \multicolumn{2}{|c|}{$\chi^{2}$ pour } \\
\hline & & & $1: 3$ & $7: 9$ \\
\hline Marianne (CVH $11 \times$ Bza 2) & 48 & 122 & 0,78 & 16,00 \\
\hline Primasol (CANP $3 \times$ Rha 266) & 56 & 154 & 0,23 & 24,21 \\
\hline Mirasol $($ Ha $89 \times$ Rha 274$)$ & 66 & 152 & 2,96 & 15,54 \\
\hline $62 \times \mathrm{ZN} 41$ & 59 & 149 & 1,08 & 19,38 \\
\hline $\mathrm{CC} 40 \times$ PAC 1 & 83 & 108 & 33,72 & 0,00 \\
\hline $\mathrm{CC} 40 \times$ PAC 2 & 61 & 144 & 2,22 & 15,75 \\
\hline $68 \times \mathrm{ZN} 41$ & 51 & 163 & 0,09 & 33,69 \\
\hline Ha $89 \times$ PA 23 & 53 & 125 & 1,92 & 13,56 \\
\hline $129 \times$ Rha 271 & 46 & 139 & 0,00 & 26,05 \\
\hline CS $511 \times$ Rha 274 & 39 & 157 & 2,45 & 44,35 \\
\hline GL $\times$ Rha 266 & 48 & 207 & 4,86 & 63,37 \\
\hline Ha $89 \times$ PR 4 & 63 & 204 & 0,21 & 43,25 \\
\hline
\end{tabular}

La F2 de "Clairsol » est particulièrement troublante : les observations sont les suivantes :

\begin{tabular}{|c|c|c|c|c|}
\hline Année & $\mathrm{ms}$ & $\mathrm{mf}$ & $1: 3$ & $\begin{array}{c}\chi^{2} \text { pou } \\
7: 9\end{array}$ \\
\hline$\overline{1979}$ & 24 & $\overline{43}$ & 3,63 & $\overline{1,40}$ \\
\hline 1981 & 67 & 144 & 4,78 & 11,85 \\
\hline $79+81$ & 91 & 187 & 8,46 & 13,26 \\
\hline 1982 & 58 & 108 & 8,22 & 4,88 \\
\hline $9+81+82$ & 149 & 295 & 16,89 & 18,33 \\
\hline
\end{tabular}

L'addition de $1979+1981$ est légitime, puisque les 2 années ne diffèrent pas $\left(\chi^{2}=0,22\right)$ de même l'addition, à ces 2 premières années, de 1982, est également légitime $\left(\chi^{2}=0,14\right)$.

En 1979, on pouvait hésiter entre les 2 hypothèses $1: 3$ et $7: 9$, par manque d'effectifs. Mais on s'aperçoit, les années suivantes, que l'augmentation des effectifs amène à rejeter l'une et l'autre des hypothèses : la proportion de ms en F2 est comprise entre 1/4 et $7 / 16$ : elle se situe à 0,33 ce qui ne correspond à aucune hypothèse génétique simple.

\section{IV. ÉTUDE DE LA GÉNÉTIQUE DE LA RESTAURATION DE FERTILITÉ SUR CYTOPLASME KOUBAN} (LECLERCQ, 1983)

On pourrait désigner par S et SK les cytoplasmes stérilisants français et Kouban.

KoUKOSH (1981) indique qu'elle a mis en évidence 19 lignées restauratrices sur cytoplasme français et 21 sur cytoplasme Kouban. Les restaurations seraient conditionnées par l'interaction de plusieurs gènes.

Le tableau 2 est constitué à partir de nos observations de 1982.

Comme pour le cytoplasme français (I.N.R.A.), il semble que la restauration s'explique le plus souvent par 1 gène dominant, parfois par 2 gènes dominants complémentaires (Bza 1, PR 4, ZN 41) qu'on peut appeler $\mathrm{Rk} 1$ et $\mathrm{Rk} 2$.

Un cas demeure mystérieux : celui de PAC 21, qui ne correspond ni à $1: 3$ ni à $7: 9$ en F2.

On pourrait étudier l'hypothèse suivante : le cytoplasme Kouban ne serait pas réellement un nouveau
TABLEAU 2

Observations de la stérilité mâle dans des descendances $F 1$ et F2 de croisements entre plantes mâle-stériles sur le cytoplasme SK (Kouban) et des lignées.

Male sterility in F1 and F2 progenies of crosses between male-sterile plants with SK cytoplasm (Kouban) and some inbred lines.

\begin{tabular}{lrrrrrr}
\hline \hline \multirow{2}{*}{ Nom du parent ${ }^{*}$} & \multicolumn{2}{c}{ F1 } & \multicolumn{2}{c}{ F2 } & \multicolumn{2}{c}{$\chi^{2}$ pour } \\
& ms & mf & ms & mf & $1: 3$ & $7: 9$ \\
\hline CCM 64 & 0 & 44 & 10 & 49 & 1,63 & 16,10 \\
Ha 89 & 0 & 42 & 50 & 151 & 0,00 & 28,33 \\
Bc 42 & 0 & 44 & 20 & 49 & 0,39 & 5,52 \\
Bza 1 & 1 & 18 & 59 & 74 & 25,56 & 0,00 \\
Bza 2 & 0 & 21 & 24 & 47 & 2,48 & 2,46 \\
PAC & 0 & 21 & 26 & 82 & 0,01 & 16,20 \\
PAC 21 & 1 & 29 & 11 & 85 & 8,68 & 39,37 \\
PAC 1 & 0 & 24 & 22 & 85 & 0,90 & 22,40 \\
PR 4 & 0 & 38 & 59 & 106 & 9,62 & 3,96 \\
ZN 41 & 8 & 13 & 35 & 38 & 19,29 & 0,36 \\
Rha 297 & 29 & 0 & & & & \\
\hline \hline
\end{tabular}

cytoplasme mais le même cytoplasme que le français, escorté de Rf1 seul, au lieu de Rf2 seul, comme c'est le cas le plus souvent avec le cytoplasme français.

Mais, dans cette hypothèse, la restauration de fertilité par le mainteneur HA 89 (S Rf1 Rf1 rf2 rf2 $\times$ rf1 rf1 Rf2 Rf2) devrait donner $7: 9$ en $F_{2}$, alors qu'on observe $1: 3$. De plus, on ne devrait pas, alors, observer la ségrégation $7: 9$ après fécondation par Bza 1, PR $4, \mathrm{ZN} 41$; en effet, nous avons attribué à ces 3 restaureurs classiques la présence de 2 dominants (Rf1 + Rf2) et les F2 issues de ces 3 restaureurs devraient donner $1: 3$ si le parent femelle portait le dominant $\mathrm{Rf} 1$. Ainsi $\mathrm{Rf}$ correspondrait à la restauration de fertilité sur cytoplasme français, Rk sur cytoplasme Kouban. Il n'est pas exclu qu'au moins 1 gène Rk s'inscrive dans la série Rf.

\section{CONCLUSION}

Le cytoplasme français, issu de $H$. petiolaris, paraît distinct du cytoplasme Kouban, mis en évidence en URSS par ANASHENKO et al. (1974) à partir de $H$. lenticularis (forme sauvage de $H$. annuus, selon certains). L'hypothèse de 2 gènes dominants complémentaires, si elle explique un bon nombre de disjonctions en $\mathrm{F}_{2}$, ne donne pas réponse à tout. Il subsiste quelques mystères. L'une de nos hypothèses actuelles est que nous n'avons pas mis en évidence, à ce stade, un mainteneur à 0 dominant $\mathrm{F} \mathrm{rf}_{1} \mathrm{rf}_{1} \mathrm{rf}_{2} \mathrm{rf}_{2}$ mais seulement des mainteneurs à 1 dominant : $\mathrm{F} \mathrm{rf}_{1} \mathrm{rf}_{1} \mathrm{Rf}_{2}$ $\mathrm{Rf}_{2}$ et que la plupart des restaureurs ont 2 dominants $\mathrm{Rf}_{1} \mathrm{Rf}_{1} \mathrm{Rf}_{2} \mathrm{Rf}_{2}$, sauf PAC 1 qui aurait un seul dominant et serait donc de type $F R_{1} R_{1} R_{1} r f_{2} \mathrm{rf}_{2}$. Pour chercher des mâle-stériles à 0 dominant, nous envisageons d'en féconder un bon nombre par PAC 1, et la $\mathrm{F}_{1}$ obtenue serait alors mâle-stérile. Pour l'étude de la stérilité mâle kouban, nous progresserons dans l'identification des gènes de restauration à partir du moment où nous aurons au moins une lignée mâle stérile homozygote, avec son mainteneur isogénique, ce qui n'est pas le cas aujourd'hui. 


\section{RÉFÉRENCES BIBLIOGRAPHIQUES}

Anashenko A. V., Mileeva T. V., Rozhkova V. T., 1974. Trudy Po Prikladnoj Botanike, Genetike i selektsii., 53 (3), 242-254.

Enns H., Dorrell D. G., Hoes J. A., Chubb W. O., 1970. Sunflower research, a progress report. Proc. 4th Int. Sunflower Conf., Memphis, Tennessee, USA, 162-167.

Fick G. N., Zimmer D. E., 1974. Fertility restoration in confectionery sunflowers. Crop Sci., 14, 603-604.

Gimenez J. D., Fick G. N., 1975. Fertility restoration of male sterile cytoplasm in wild sunflowers. Crop Sci, 15, 724-726.

Kinman M. L., 1970. New developments in the USDA and State Experiment Station sunflower breeding programs. Proc. 4th Int. Sunflower Conf., Memphis, Tennessee, USA, 181-183.

Koukosh Marie V., 1981. Some aspects of a study of fertility restorers in sunflower (Helianthus annuus L.) (en russe). Byull. Vses. Int. Rastenievodstva Imeni N.I. Vavilova, 115, 44-49.

Leclercq P., 1969. Une stérilité mâle cytoplasmique chez le tournesol. Ann. Amélior. Plant., 19 (2), 99-106.
Leclercq P., 1971. La stérilité mâle cytoplasmique du tournesol. I. Premières études sur la restauration de fertilité. Ann. Amélior. Plant., 21 (1), 45-54.

Leclercq P., 1979. La stérilité mâle cytoplasmique du tournesol. II. Influence de la génétique et du milieu sur la restauration de la fertilité. Ann. Amélior. Plant., 29 (2), 201-212.

Leclercq P., 1983. Etude de divers cas de stérilité mâle cytoplasmique chez le tournesol. Agronomie, 3 (2), 185-187.

Reddy P. S., Thammi Raju B., 1977. Inheritance of fertility restoration in sunflower (Helianthus annuus L.). Euphytica, 26, 409-412. 409-412.

Vranceanu V. A., Stoenescu F. M., 1971. Pollen fertility restorer gene from cultivated sunflower (Helianthus annuus L.). Euphytica, 20, 536-541.

Vranceanu V. A., Stoenescu F. M., 1978. Genes for pollen fertility restoration in sunflowers. Euphytica, 27, 617-627. 